\title{
Exploration and Practice of New Material Technology Hatching System
}

\author{
Xinsheng Zhao ${ }^{1, \text { a }}$ \\ ${ }^{1}$ Jiangxi University of Applied Science,Nanchang,Jiangxi,China,330100 \\ '179059647@qq.com
}

\begin{abstract}
In our age, all progress has been made, thanks to the constant explorations of our forefathers on the road to development. The progress of this age can be from the ancient stone age to the bronze and iron age to the present information and carbon age. From this, we can see that the material science and technology are closely related to the times, but also promote the progress of human civilization, new materials are the basis of scientific and technological innovation of the times, there is a special significance.
\end{abstract}

Keywords: incubation system; new materials; scientific innovation

\section{新材料科技孵化体系的探索与实践}

赵新胜 ${ }^{1, a}$

${ }^{1}$ 江西应用科技学院, 南昌, 江西, 中国, 330100

a179059647@qq.com

\section{摘要}

在我们所处的这个时代，一切都得以进步，得益于先辈在发展的道路上，不断的探索着。这种时代的 进步可以具体为从远古的石器时代到青铜、铁器时代, 再到现在的信息、碳素时代。而从这当中可以 看出材料科技与时代有着至关密切的联系，同时也推动着人类文明的进步，新的材料是时代科技创新 的依据, 存在着非凡的意义。

关键词：䁔化体系；新材料；科技创新

\section{1. 睬化器推动新材料产业的发展}

企业对于新材料的研究要形成专门研究人员, 使其形成 共同发展体系。

\section{1 新材料当今状况}

到目前为止, 新材料产业发展已经有所成果, 其产 值将达到 2 万亿元。这也使得高校的重视, 从而开展以 新材料的研究课程, 据世界大学研究数据表明, 我国有 五所高校对于新材料的研究排到世界高校的前五十。在 沿海地区对新材料科技研究更为注重, 而中西部地区则 打造出特色鲜明的新材料产业基地。

由此看出我国对于新材料的注重, 科技和产业两方 面来看, 我国是具有一定的发展基础的。与其他国家对 比, 还是存在着很多问题, 由于对其研究的落后导致积 累的经验不够丰富, 创新能力还不够强大, 原创性的东 西太少，从而导致核心技术成为别人的专利。国内的对 其研究大多数为实验室主, 而其研究主要是解决材料的 有无问题, 并不全面, 对其工程, 生产等方面不重视, 这些点归根结底还是研究团队的不足, 研究经验无法相 互学习相互进步。针对这些研究人员要集中在某区域内,

\section{2 殍化器对于新材料的作用}

所研究的材料要能在现实中体现其价值来, 在创新 出新材料到实际运用推广中, 需要有三个必要的阶段。 首先是在实验室研究的过程中, 研究人员要要有着明确 的设计理念、研究的方向, 然后将其设计理念运用与实 际, 研究出样品, 研究所则需要对样品进行模拟环境测 试验证, 使其达到用户的要求及肯定, 研究好产品后则 需要工厂统一生产, 生产之后并不是大功告成, 需要对 其产品不断跟踪优化, 确保成为货架产品。这是一件产 品研究发展到生产的必要过程, 在这过程中也是存在着 一定的风险性。

在新材料的成为货架产品周期中，需要经过政府的 许可, 及各种利益方人员的参与, 而在中小型的企业中, 则会存在着资金紧缺，开发技术不成熟，进入市场的困 难, 而面对这些存在的困难, 企业不能解决, 这时候则 需要孵化器来作为载体, 解决这些所存在的问题。 


\section{2. 我国孵化器的发展现状及问题}

\section{表 1 我国孵化器的发展现状及问题}

国内企业对于孵化器的专业服务存在依赖性, 使得孵 化器开始朝着孵化专业服务的方向发展, 虽然孵化专 业服务得以快速发展，但是企业对其只是存在性依 赖, 并不能带动企业团队自主发展, 使其企业创新仍 存在原地踏步阶段。

\begin{tabular}{|c|c|c|}
\hline $\begin{array}{c}\text { 孵化管理模式 } \\
\text { 经验缺乏 }\end{array}$ & $\begin{array}{c}\text { 软硬件服务能 } \\
\text { 力欠缺 }\end{array}$ & $\begin{array}{c}\text { 专业管理模式 } \\
\text { 经验不足 }\end{array}$ \\
\hline $\begin{array}{l}\text { 以目前形式而 } \\
\text { 论, 国内的孵化 } \\
\text { 器主要来源还 } \\
\text { 是政府, 对于这 } \\
\text { 种单一的存在, } \\
\text { 市面上没有竞 } \\
\text { 争的压力, 导致 } \\
\text { 其服务质量难 } \\
\text { 以发提升, 服务 } \\
\text { 水平停留不前。 }\end{array}$ & $\begin{array}{l}\text { 国家仍处在发 } \\
\text { 展阶段, 导致需 } \\
\text { 要科技还不是 } \\
\text { 很全面, 而由政 } \\
\text { 府所提供的孵 } \\
\text { 化器, 也只能解 } \\
\text { 决点硬件上的 } \\
\text { 问题, 对于核心 } \\
\text { 技术的掌握还 } \\
\text { 是存在着问题 } \\
\text { 的, 在孵化的过 } \\
\text { 程中难以帮助 } \\
\text { 企业解决各类 } \\
\text { 问题, 突破难 } \\
\text { 关。 }\end{array}$ & $\begin{array}{l}\text { 针对我国现有 } \\
\text { 的孵化器, 大多 } \\
\text { 数是政府的管 } \\
\text { 理, 而这种单一 } \\
\text { 的管理模式, 会 } \\
\text { 容易导致企业 } \\
\text { 的发展得不到 } \\
\text { 进步, 设计的周 } \\
\text { 期得不到提升 }\end{array}$ \\
\hline
\end{tabular}

企业孵化器通俗大体的来说就是对中小型技术企 业进行培育为其提供服务的机构。在中小型创业起步阶 段中, 孵化器能很好的为企业提供一系列的服务, 比如 创业的基础设施与资源, 给企业起步阶段作个引导, 大 大的提高创业的成功率, 也为其减少一些没必要的开销, 从而达到推动科技进步的转化。而在国内企业对于孵化 器的专业服务存在依赖性, 使得孵化器开始朝着孵化专 业服务的方向发展，虽然孵化专业服务得以快速发展， 但是企业对其只是存在性依赖, 并不能带动企业团队自 主发展，使其企业创新仍存在原地踏步阶段。

\section{1 瞬化管理模式经验缺乏}

以目前形式而论, 国内的孵化器主要来源还是政府, 对于这种单一的存在，市面上没有竞争的压力，导致其 服务质量难以发提升, 服务水平停留不前。在这阶段要 也出现第三方的孵化器, 由于收益方面还没有明确的成 果, 而孵化器帮助企业的周期长, 孵化器的管理模式还 是需要不断地探究。

\section{2 软硬件服务能力欠缺}

国家仍处在发展阶段，导致需要科技还不是很全面，
而由政府所提供的孵化器, 也只能解决点硬件上的问题, 对于核心技术的掌握还是存在着问题的，在孵化的过程 中难以帮助企业解决各类问题，突破难关。

\section{3 专业管理模式经验不足}

针对我国现有的孵化器, 大多数是政府的管理, 而 这种单一的管理模式, 会容易导致企业的发展得不到进 步，设计的周期得不到提升。而解决这事则需要引进专 业的管理体系，设定一套合理的管理体系，从而能帮助 企业的管理模式，减少企业孵化的周期。而帮助企业孵 化过程中并不是简单的交流对接, 这是需要确定一套完 善的体系的, 要确保从理论的思想到实际操作研发到检 测产品, 到最后的市场推广的科技成果转化。而在这种 孵化的过程中包含着人才，技术，管理模式等方面的因 素转化。而国内孵化现状还是远远达不到的。针对这种 孵化模式首先需要做到一下几点。

\section{3. 1 科技成果的市场化评估}

科技成果的转化作为重要的环节, 往往最容易得到 忽略，科技的成果并不能一方的测试肯定的，这是不可 行的, 科技成果转化需要专业人士和孵化机构的认可, 并对成果进行市场价值评估。

\section{3.2 企业有效科技需求的收集}

企业对于自己所需求的不够明确，企业与企业之间 的现状不够了解等因素阻碍企业发展, 针对现状企业应 对实地研究对于企业本身所需求的技术与孵化机构合 作的模式发展探索, 从而达到理想科技成果转化。

\subsection{3 科技成果与企业需求相匹配}

建立专业团队，收集科技成果，针对企业所需要的 科技成果，进行有效的匹配对接。高效率的完成科技成 果与企业需求。

\section{3. 4 供到核心技术的服务}

对于现仅有的办公政策等基础设施服务中，要不断 的学习引进专业化的管理模式, 做到能为其提供到核心 技术的服务。 


\section{3. 创新的新材料孵化器模式}

\section{1 新材料产业化过程}

科技成果的孵化要得到创新需要经历各方面的创 新, 而在科技成果创新中, 新产品生产模式则需要发生 改变, 首先所设计的科技需求需要满足可持续发展的社 会要求, 专业化人才解决设计的核心性问题, 同时孵化 授权企业, 帮助企业进行孵化, 企业对于产品则进行开 发, 设计出样品, 并对样品验证测试, 确保无误联系厂 家进行生产, 生产出来的产品孵化机构帮助寻找市场及 初期运营阶段，最后投放的市场，满足市场需求。

\section{2 科技成果创新餒化完善体系}

而要做到新产品产业化孵化, 以上的方式还是存在 着欠缺的, 而针对上面的模式, 则需要建立三个相互独 立的模式, 这种共存依赖的模式构建成完整生态型科技 创新链。以下将一一描述: 首先, 市场模块, 市场根据 社会所需求进行明确的方向, 保证所研究的能满足社会 需求。其次, 科学家模块, 科学家需要与企业进行明确 的对接, 孵化包含技术, 人才培养, 资金问题, 政策管 理, 信息交接, 空间储存, 设施市场等一系列要素的对 接, 确保人才孵化的独立性, 培养其团队合作能力, 对 于核心问题作到指导性思想, 拓阔企业自身实力, 帮助 企业有效的发展。最后成果转化模块, 科学家的研究成 果要与企业之间作到完善的交接, 企业对于成果, 要建 立专业人才管理, 收集成果并对其有效的处理, 待企业 有所需求时能准确地进行衔接, 从而达到成果与企业之 间真正的对接。创新孵化链则能高效率的帮助企业进行 长远的发展。

\section{4. 科技睬化器未来发展趋势}

科技企业孵化器经过三十多年的发展, 已经进入新 的历史时期, 在以科技驱动创新的战略国策下, 它必须 承担更重要的责任, 也必须实现自身的进化。从未来发 展角度来看, 科技企业孵化器需要在资源层面, 能力层 面以及价值层面应对新的挑战与新的变化。

\section{1 资源层面}

科技捊化器是创新资源聚集的平台, 未来的资源聚 集应以共享、国际化、区域化和信息化的方式发展。简 而言之, 共享意味着创新要素, 包括人员、设备、能力 和信息, 可以通过孵化器平台共享。国际化意味着打破 国界, 真正实现资源层面的全球资源积累和配置。区域 化意味着在特定区域, 特定领域要有特色定位, 不能千 遍一律; 信息化是指互联网、物联网和人工智能等新一 代信息技术，它将给资源的汇集和优化配置带来巨大变
化。

\section{2 能力层面}

回归自然, 科技孵化器需要具备三个核心能力: 判断力、整合性和转化性。判断力是指判断技术、市 场、商业模式和创新团队的能力; 整合性是指围绕技 术和项目整合各类创新资源要素, 优化配置形成合 力; 转化性是指孵化器在技术与产业之间架起一座桥 梁, 并且在连接过程中实现各类创新资源和要素的价 值体现。

\section{3 价值层面}

科技孵化器不应仅仅是培育和帮助高新技术企业 的服务机构。从技术和产业两个层面, 体现捊化器的价 值, 最终应该在供给端实现科技成果产业化, 实现企业 需求的技术升级改造。

\section{5. 结语}

通过以上分析, 当今现状的孵化器存在着很多的漏 洞与不当，没有竞争压力。单一的孵化模式对于企业来 说, 只能提供某些硬件服务方面的帮助, 实际上, 很难 起到决定性的重大帮助。而针对这些不足之处，应该建 立新型孵化链, 从科技需求到人才运用管理, 到帮助企 业得到技术解决方案, 再到市场销售等方面的完善孵化 体系管理模式。这些不仅仅提供硬件服务，还可以为企 业带来软件等方面的其他服务, 帮助企业更好的孵化出 属于自己企业的人才, 更为重要的是能够提供更好的科 技成果转化的效果。

\section{References}

[1] Naicheng Cao. Case and Analysis of Technology Risk for New Material Enterprises [J]. New Materials Industry ,2009,(12):47-48.

[2] Du Debin. Comprehensive and Objective Understanding of the Transformation of Scientific and Technological Achievements in Colleges and Universities in China [N] . Guangming Daily ,2015-1212(07).

[3] Guo Qiang, Xia Xiangyang, Zhao Li. A Study on Influencing Factors and Countermeasures of Transformation of Scientific and Technological Achievements in Colleges and Universities [J] . Science and Technology Progress and Countermeasures ,2012,29(6):151-153. 
[4] Mei Mei E, Zhong Weijun. Analysis on the Factors of Impediments to the Transformation of Scientific and Technological Achievements in Colleges and Universities in China [J]. Science and Technology Management ,2008(3):22-27.

[5] Du Weijin, Song Yuan, Li Jing, et al. Evolution of Science and Technology Transformation Policy and Analysis of Regional Differences: A Case Study of Beijing-Tianjin-Hebei and Yangtze River Delta [J]. Science and Technology Management ,2017,38(2):3-11.

[6] Ma Jiangna, Li Hua, Wang Fang China Science and Technology Achievement Transformation Policy Text Analysis: Based on Policy Tools and Innovation Value Chain [J]. Science and Technology Management Research ,2017(7):34-42.
[7] Liuliu, Wanghua, Guijuan Wang. Construction of Incubation System of Science and Technology Enterprises in Tangshan City[J]. The Think Tank Age. 2019(32):30-31.

[8] SuFang Qi. How to Break through the Construction of Urban Science and Technology Innovation Service System[J]. East China Science and Technology. 2019(07):52-59.

[9] Liuhui, Jiawei Liu, Jingyi Wang, Wangtao. Summary of Research on Evaluation Index System and Methods of Science and Technology Service[J]. Standard science. 2019(03):18-24.

[10] Yangliu, Huangying, Liutong. Research on the Evaluation System for the Incubation of Scientific and Technological Achievements[J]. Research on Modern State-owned Enterprises. 2019(04):103-105. 